\title{
problème des fissures sur les murs des bâtiments du monastère de Stavronikita du mont Athos (Grèce)
}

\author{
C.A. DEMIRIS \\ J.D. MELADIOTIS
}

Laboratoire de Géologie de l'Ingénieur

Université Aristote de Thessalonique

\section{INTRODUCTION}

Le monastère de Stavronikita dont l'histoire commence à partir du $\mathrm{X}^{e}$ siècle, est situé à peu près au milieu de la côte septentrionale de la péninsule du mont Athos, branche orientale de la presqu'île de la Calcidique au Nord de la Grèce. Il est fondé sur une masse rocheuse très escarpée, à $42 \mathrm{~m}$ au-dessus du niveau de la mer (fig. 1).

Le plus vieux bâtiment du monastère est celui du "Pyrgos" qui a été bâti au début du $\mathrm{XI}^{e}$ siècle. Le "Pronaos" a été bâti un siècle plus tard. Les trois contreforts de la côte orientale du monastère ont été construits en 1842 tandis que les bâtiments qui s'appuient sur ces contreforts étaient érigés en 1883.

Le problème principal du monastère est celui de sa stabilité, en raison de la présence d'un grand nombre de fissures sur les murs de ses bâtiments qui se développent suivant deux lignes sensiblement horizontales et qui s'élargissent pendant l'hiver ou à l'occasion de puissantes secousses sismiques.

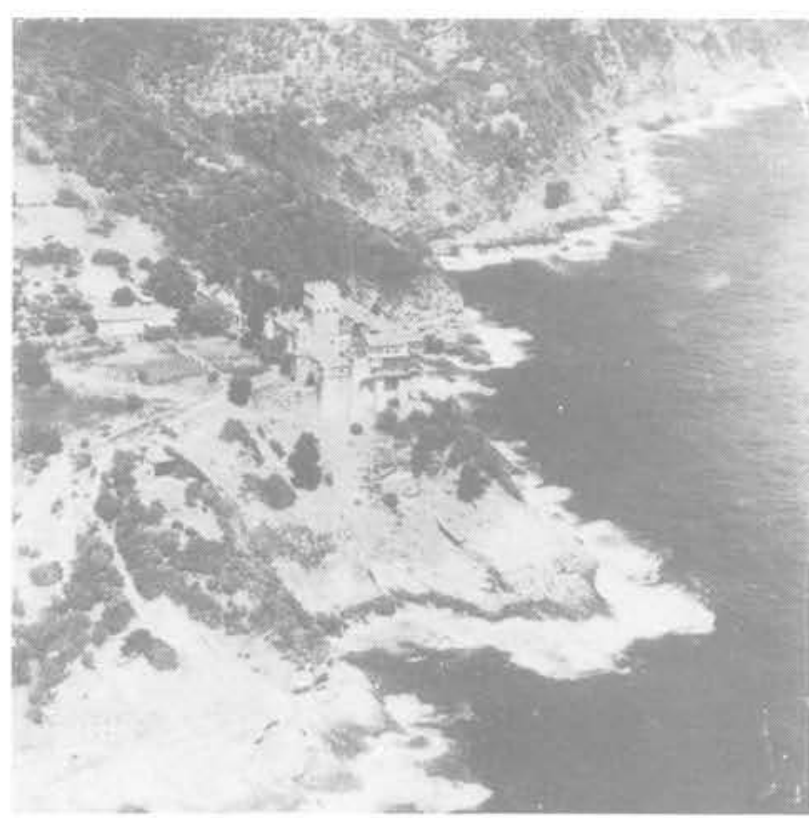

Fig. 1. - Le monastère de Stavronikita. 
Ces fissures qui ne sont dues ni à une mauvaise fondation des bâtiments, ni au tassement différentiel du sous-sol, proviennent en fait d'un déplacement horizontal de quelques centimètres, du compartiment oriental du monastère par rapport au compartiment occidental supposé immobile, visible sur la côte Nord (fig. 2).

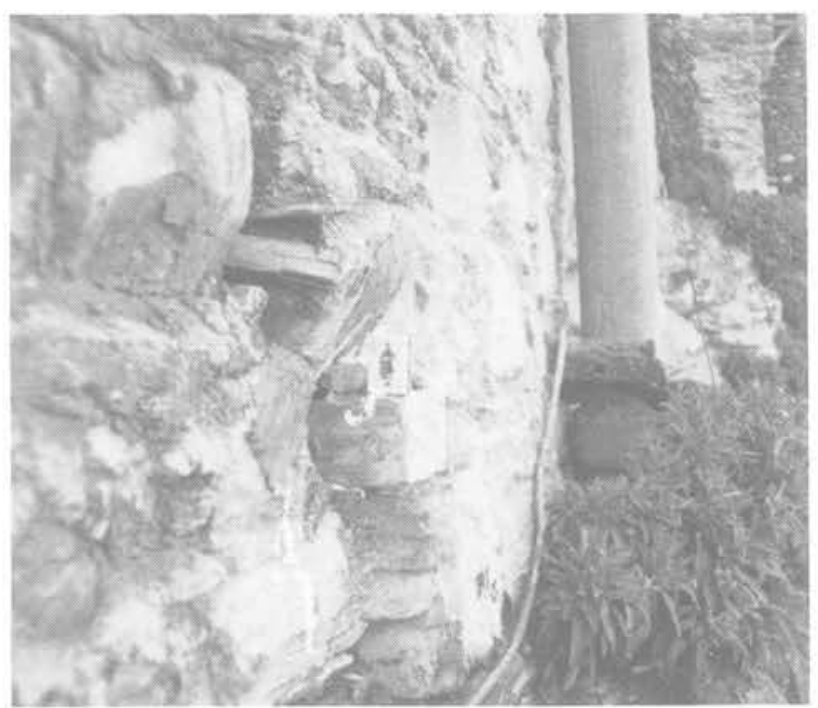

Fig. 2. - Fissures du mur du monastère provoquées par un déplacement horizontal.

Le déplacement du compartiment oriental du monastère vers le Sud, est dû au glissement de la masse rocheuse sous-jacente, qui lui sert de fondation.

Le but essentiel de cette étude est de préciser les modalités de glissement, la géométrie et les conditions d'équilibre de la masse rocheuse glissante.

\section{CADRE GÉOLOGIQUE}

\subsection{Situation géologique de la péninsule du mont Athos}

La péninsule du mont Athos, prolongement SE du massif Serbomacédonien, doit sa morphologie aux ruptures et aux effondrements de l'Eghis au tertiaire et au quaternaire.

Au point de vue géologique, la péninsule est constituée, de bas en haut:

- par un socle cristallin et cristallophyllien paléozoique, ou plus ancien, formé de gneiss, d'amphibolites et de péridotites,

- par une masse crystallophyllienne paléozoïque très plissée qui est constituée de bas en haut par:

- la série de Kerdilia, formée de calcaires cristallins alternant avec des gneiss ceillés,

- la série de Vertiskos, d'âge hercynien, formée de micaschistes, de gneiss et d'amphibolites,
- par un horizon cristallin et cristallophyllien mésozoïque, formé de granites à biotite et de gneiss à plagioclase,

- par les calcaires cristallins triasiques de l'unité d'Aspri Vryssi-Chortiatis.

\subsection{Situation géologique de la région étudiée}

Les formations géologiques du monastère de Stavronikita dont les caractéristiques ont été déterminées au Laboratoire de l'Ecole polytechnique de l'Université Aristote de Thessalonique, appartiennent au socle cristallophyllien et contiennent les plans, en deux unités, lithologiques et stratigraphiques suivants:

- l'unité inférieure des leptynites;

- l'unité supérieure qui comprend les gneiss à deux micas, les gneiss à mica noir et les amphibolites.

Les gneiss à deux micas ( $(\zeta 2 \mathrm{~m}$ ) forment le support sur lequel est fondé le monastère de Stavronikita. Ce sont des roches sombres, à grain moyen, plus ou moins quartzeux - 35-55\% - feldspathiques - 26-49\% - et micacés - 23-27\% - dont la teneur en biotite peut varier entre 0 et $15 \%$. Les gneiss à deux micas qui présentent une schistosité très nette et une altération superficielle très intense, donnant aux affleurements une teinte brune à jaunâtre, sont parcourus par des filons quarzitiques qui se développent en général parallèlement à la stratification.

Les gneiss à mica noir ( $\zeta$ bi) se présentent sous forme d'un banc mince intercalé entre les gneiss à deux micas et les leptynites sous-jacentes. Ce sont des roches schisteuses, à grain fin, noires, formées de biotite - $35 \%-$, de feldspaths $-37 \%-$, de quartz $-20 \%$ - et de muscovite $-8 \%$.

Les amphibolites (am) sont en fait des schistes cristallins vert foncé, très durs, où domine l'amphibole $57 \%$ - associée aux feldspaths - $19 \%$ - à l'épidote $-15 \%$ - et au quartz - $9 \%$.

Les leptunites $(\lambda)$ sont claires, blanchâtres, à grain fin, pauvres en éléments noirs. Elles sont compactes et dures, formées de quartz - 50\% - de feldspath $30 \%$ - de muscovite - $15 \%$ - de sillimanite $3 \%$ - et de biotite - $2 \%$.

\subsection{Remarques tectoniques et structurales}

Au point de vue tectonique, la zone étudiée est marquée par l'existence d'un pli couché à l'échelle métrique visible en coupe perpendiculaire à l'axe au talus septentrional du monastère.

Le rapport des épaisseurs orthogonales du pli couché $-t^{\prime}=0,3$ - et de l'angle $\alpha-\alpha=80^{\circ}$ - conduit au diagramme de classification de Ramsay et montre qu'il s'agit d'un pli semblable dont le plan axial se trouve à une hauteur de 2 mètres au-dessus du niveau de la mer, avec une orientation $\mathrm{N} 90^{\circ}-\mathrm{S} 15^{\circ}$ 
Les formations cristallophylliennes qui ont été soumises aux plissements, sont accompagnées par un clivage schisteux $\left(S_{1}\right)$ qui a débité les roches en minces feuillets. Dans les gneiss à deux micas et les gneiss à mica noir qui sont incompétents, le clivage schisteux est très serré, parfaitement réglé et souligné par les recristallisations des minéraux dans le plan axial du pli couché. Par contre, dans les leptynites compétentes, le clivage schisteux est très écarté et converge vers le plan axial du pli couché.

Les accidents tectoniques de la région se manifestent sous forme de failles normales réparties en deux régimes principaux (fig. 3 ):

- un régime initial des grandes failles $\left(F_{1}\right)$ parallèles entre elles, de direction EW:

- un régime des failles secondaires $\left(F_{2}\right)$ de direction subverticale, qui forment avec les failles précédentes des blocs parallélépipédiques dont la grande arête est dirigée vers l'Est.

Les failles $\left(F_{1}\right)$ se manifestent sous forme de marches d'escalier et constituent un fossé d'effondrement annexe entre deux petits horst à l'endroit du cimetière et du stavros. Le rejet vertical maximum de ces failles qui se développe au talus septentrional du cimetière, est de l'ordre de $20 \mathrm{~m}$. Par contre, à l'endroit du stavros, les rejets verticaux des failles sont de l'ordre de quelques décimètres et s'accompagnent, dans les gneiss à mica noir incompétents, de microplis à Péchelle décimétrique.
L'existence des boudinages dans les gneiss à deux micas et les gneiss à mica noir - bancs quartzitiques compétents intercalés entre les niveaux des gneiss incompétents - montre que le clivage schisteux est parallèle à leur stratification où se produit l'étirement maximum (fig. 4a).

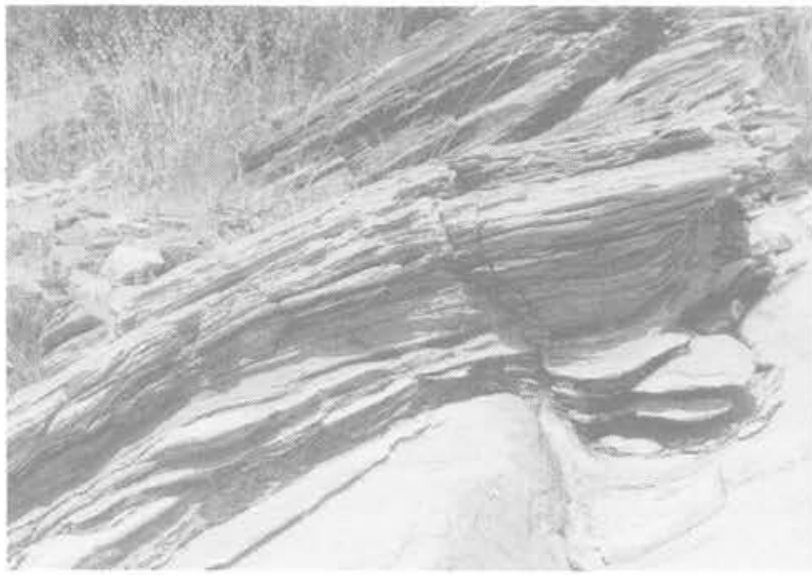

Fig. 4(a). - Schistosité du massif.

Les flancs du pli couché restant parallèles au plan axial, on peut représenter la structure de l'ensemble de la région étudiée comme un panneau monoclinal faillé, incliné vers le SE.

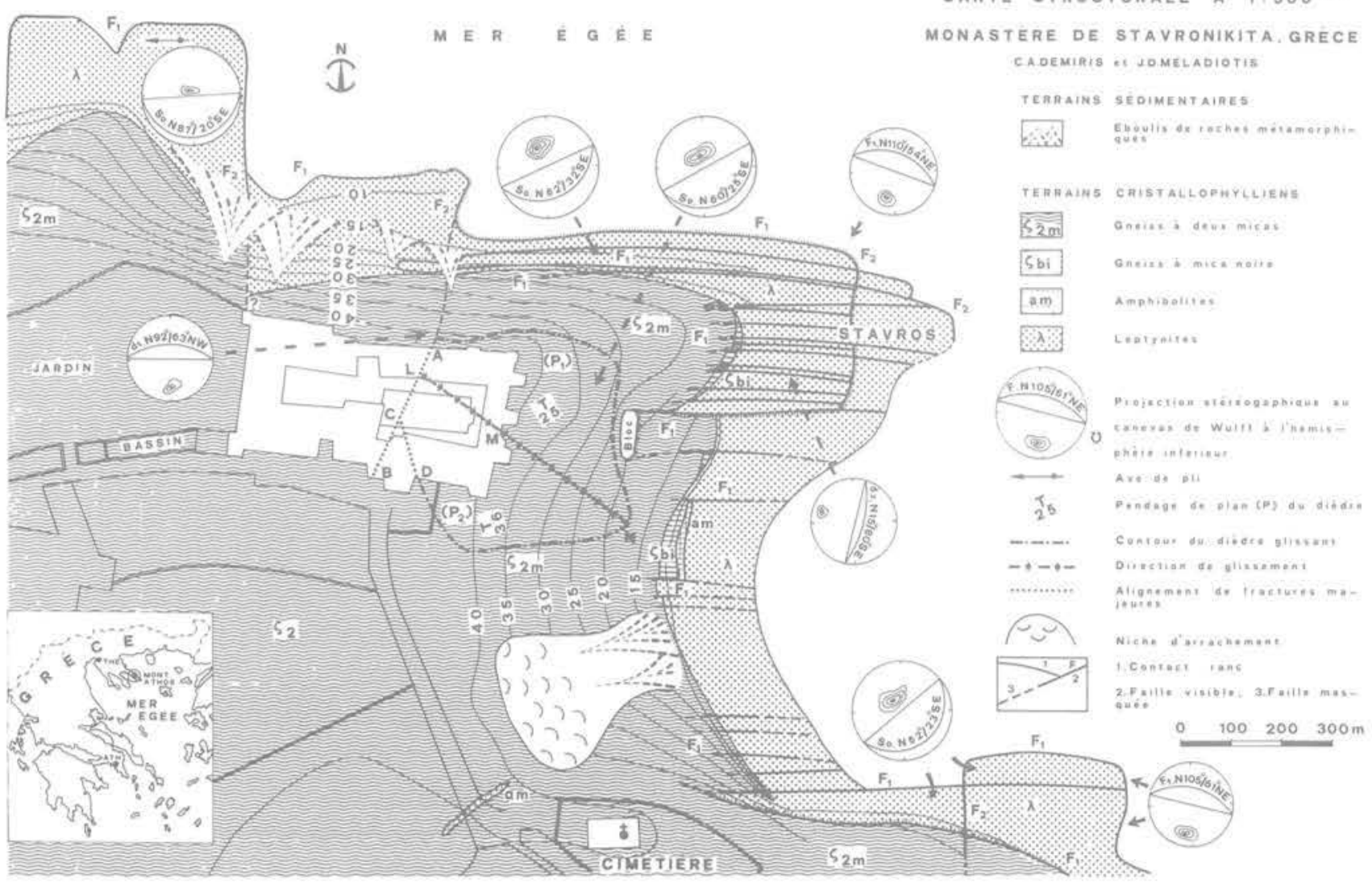

Fig. 3. - Carte des accidents tectoniques. 


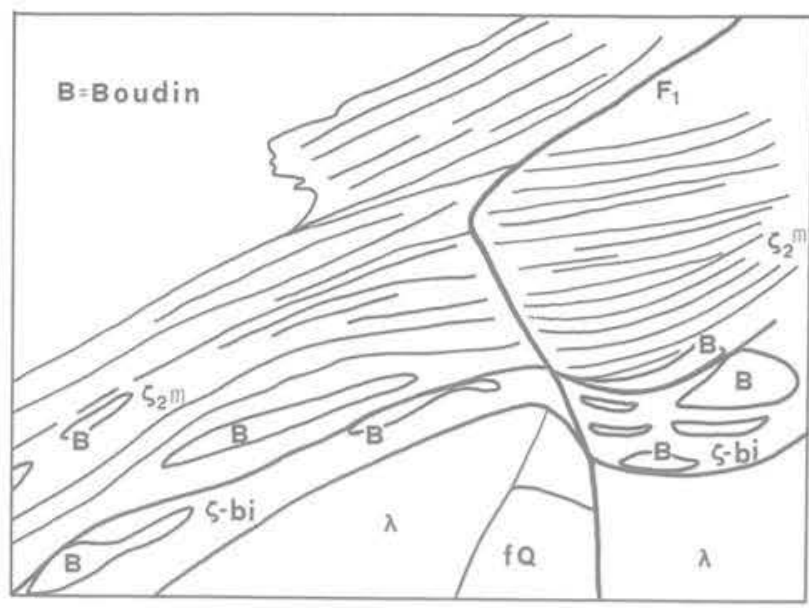

Fig. 4(b). - Croquis d'interprétation. $\left(\zeta_{2} m\right)$ gneiss à deux micas (ג) leptynite (ל-bi) gneiss à mica noir
(fQ) filons de quartz (B) boudin $(F$,$) faille$

\section{MESURES IN SITU ET EN LABORATOIRE}

Pour mettre en évidence les causes des fissures des murs du monastère, plusieurs mesures in situ et en laboratoire ont été effectuées pour déterminer les paramètres du massif de fondation.

\subsection{Orientation des plans de stratification et de discontinuité des formations géologiques}

Pour déterminer l'orientation des plans de stratification et des plans de discontinuité des formations géologiques, 400 mesures ont été réalisêes sur les talus rocheux du monastère.

A partir des diagrammes stéréographiques des mesures sur l'hémisphère inférieur du canevas de Schmidt, on a obtenu les résultats suivants.

3.1.1. Sur le talus oriental du monastère, l'orientation du plan de stratification des gneiss à deux micas $-N$ $60^{\circ}$-SE $25^{\circ}-$, coïncide avec l'orientation du plan de stratification des leptynites qui est $\mathrm{N} 62^{\circ}$-SE $32^{\circ}$.

La différence d'orientation des plans de stratification des leptynites entre la zone ci-dessus et celle du talus septentrional du monastère - N $87^{\circ}$ - SE $20^{\circ}$ - est due au basculement du horst du stavros vers l'Est.

3.1.2. L'orientation des plans de failles $\left(F_{1}\right)$ dans les leptynites varie entre $\mathrm{N} 110^{\circ}-\mathrm{NE} 54^{\circ}$ sur le talus septentrional du monastère et $\mathrm{N} 105^{\circ}-\mathrm{NE} 61^{\circ}$ sur le talus septentrional du cimetière.

3.1.3. L'orientation des plans des failles secondaires $\left(\mathrm{F}_{2}\right)$ varie entre $\mathrm{N} 0^{\circ}-90^{\circ}$ et $\mathrm{N} 20^{\circ}$-SE $85^{\circ}$.
3.1.4. Le plan majeur des diaclases $\left(d_{1}\right)$ dans les gneiss à deux micas sur le talus septentrional du monastère, a une orientation $\mathrm{N} 92^{\circ}-\mathrm{W} 63^{\circ}$

3.1.5. L'orientation du plan majeur des diaclases $\left(d_{2}\right)$ dans les leptynites sur le talus oriental du monastère est $\mathrm{N} 15^{\circ}$-SE $80^{\circ}$.

\subsection{Direction du déplacement du compartiment oriental du monastère}

Pour mettre en évidence la direction de déplacement du compartiment oriental du monastère, plusieurs mesures ont été effectuées sur les 21 points exacts de fractures majeures qui se développent, comme on l'a vu précédemment, le long de deux lignes horizontales sécantes $A B$ et $C D$ (fig. 3 ), en déterminant chaque fois la grandeur et la direction de leurs rejets horizontaux, à partir des coordonnées $\kappa$ et $\mu$ de la figure 5 .

Les mesures ont êté exécutées près de la fondation des bâtiments où les écartements entre les lèvres des fractures sont les plus larges.

A partir des résultats des mesures précédentes, on constate que le déplacement du compartiment oriental du monastère, par rapport au compartiment occidental supposé immobile, est représenté par la ligne brisée ACD et se produit le long de la ligne horizontale LM dont la direction est $\mathrm{N} 126^{\circ}$ (fig. 3)

\subsection{Angle de frottement interne des gneiss à deux micas}

A partir d'échantillons secs et d'échantillons saturés d'eau, l'angle de frottement interne des gneiss à deux micas a été déterminé au laboratoire, d'une part parallèlement au plan de leur stratification et d'autre part, perpendiculairement à ce plan.

Le résultat de ces essais réalisés au Laboratoire de l'Ecole polytechnique de l'Université Aristote de Thessalonique, est donné au tableau I.

Tableau I

\begin{tabular}{|c|c|c|c|c|c|}
\hline \multicolumn{3}{|c|}{ Echantillon sec } & \multicolumn{3}{|c|}{ Echantillon saturé d'eau } \\
\hline \multirow{2}{*}{$\begin{array}{c}\begin{array}{c}\text { Contrainte } \\
\text { normale } \\
\text { (supposée } \\
\text { uniforme) }\end{array} \\
\text { N }\end{array}$} & \multicolumn{2}{|c|}{$\begin{array}{c}\text { Angle } \\
\text { de } \\
\text { frottement } \\
\text { interne }\end{array}$} & \multirow{2}{*}{$\begin{array}{c}\begin{array}{c}\text { Contrainte } \\
\text { normale } \\
\text { (supposée } \\
\text { uniforme) }\end{array} \\
\mathrm{N}\end{array}$} & \multicolumn{2}{|c|}{$\begin{array}{c}\text { Angle } \\
\text { de } \\
\text { frottement } \\
\text { interne }\end{array}$} \\
\hline & $\operatorname{tg}$ & $\varphi$ & & $\operatorname{tg}$ & $\varphi$ \\
\hline \multicolumn{6}{|c|}{$\begin{array}{l}\text { SURFACE PARALLÈLE AU PLAN } \\
\text { DE STRATIFICATION (So) en MPa }\end{array}$} \\
\hline $\begin{array}{l}0,1 \\
1,0\end{array}$ & $\begin{array}{l}0,54 \\
0,51\end{array}$ & $\begin{array}{l}27^{\circ} \\
28^{\circ}\end{array}$ & $\begin{array}{l}0,1 \\
1,0\end{array}$ & $\begin{array}{l}0,42 \\
0,42\end{array}$ & $\begin{array}{l}23^{\circ} \\
23^{\circ}\end{array}$ \\
\hline \multicolumn{6}{|c|}{$\begin{array}{l}\text { SURFACE PERPENDICULAIRE AU PLAN } \\
\text { DE STRATIFICATION (So) en MPa }\end{array}$} \\
\hline $\begin{array}{l}0,1 \\
1,0\end{array}$ & $\begin{array}{l}0,62 \\
0,61\end{array}$ & $\begin{array}{l}32^{\circ} \\
31^{\circ}\end{array}$ & $\begin{array}{l}0,1 \\
1,0\end{array}$ & $\begin{array}{l}0,50 \\
0,52\end{array}$ & $\begin{array}{l}26,5^{\circ} \\
27,5^{\circ}\end{array}$ \\
\hline
\end{tabular}




\section{CONDITIONS D'ÉQUILIBRE EXISTANTES}

\subsection{Conditions de glissement}

La découverte de glissement sur le talus oriental du monastère, confirme notre opinion initiale d'après laquelle le déplacement du compartiment oriental du monastère est dû au glissement de la masse gneissique sous-jacente.

Plus précisément, dans les gneiss à deux micas du talus rocheux ci-dessus, entre les courbes de niveau 25 et $30 \mathrm{~m}$ et à côté du bloc parallélépipédique indiqué sur la carte de la figure 3 , on a déterminé sur un plan N $5^{\circ}$-SE $83^{\circ}$, une zone de cisaillement senestre, c'est-à-dire une zone des discontinuités conjuguées correspondant aux fissures de Riedel.

A partir des dièdres aigus des lentilles de cisaillement qui se forment par les fissures de Riedel et les plans de cisaillement, on détermine un plan de glissement $\left(\mathrm{P}_{1}\right)$ dont l'orientation s'identifie avec le plan de stratification des gneiss à deux micas.

Si le déplacement du compartiment oriental du monastère (fig. 5) est dû au glissement précité, la direction de déplacement du compartiment oriental du monastère - N $126^{\circ}$ - aurait dû s'identifier avec la direction de pendage du plan de glissement - N $150^{\circ}$ mais, d'après les données ci-dessus, ce n'est pas le cas.

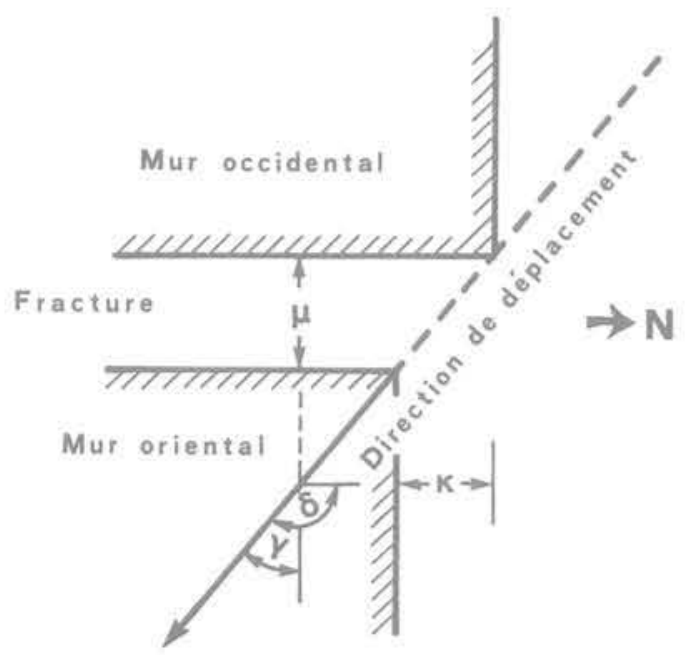

Fig. 5. - Direction des déplacements du monastère.

Puisque la direction de la ligne horizontale $A B$, sur laquelle se développe un grand nombre des fractures majeures aux murs des bâtiments du monastère, coïncide avec la direction du plan de la faille $\mathrm{F}_{2}-\mathrm{N}$ $20^{\circ}$-SE $85^{\circ}$ - qui traverse le talus septentrional du monastère, la faille doit passer au-dessous du monastère.

En outre, puisque une grande partie des fractures majeures des murs des bâtiments du monastère se développe le long de la ligne horizontale $\mathrm{CD}$, comme on l'a déjà vu, on suppose que les fractures ci-dessus proviennent d'un deuxième plan de cisaillement $\left(\mathrm{P}_{2}\right)$ dont la direction doit coincider avec la direction de la ligne $\mathrm{N} 165^{\circ}$ (fig. 6).

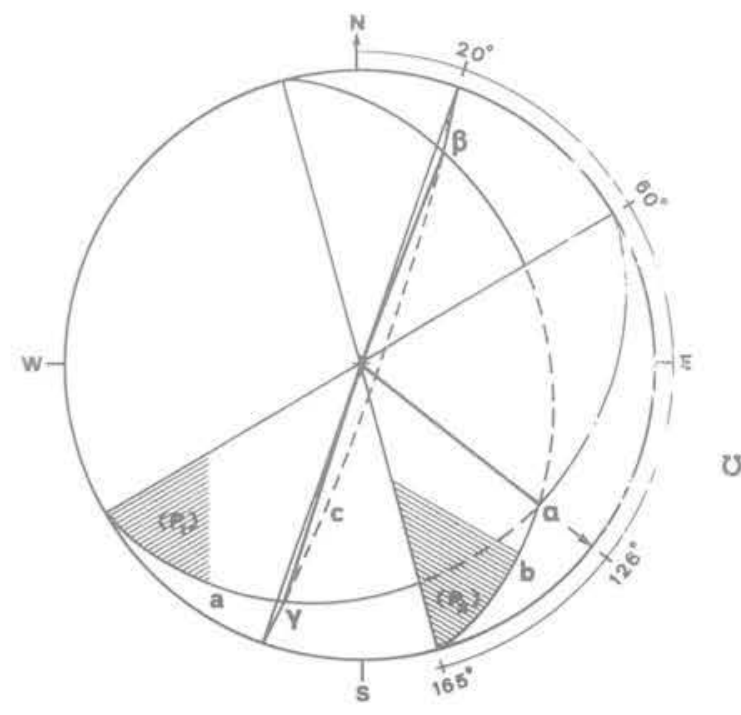

Fig. 6. - Orientation des failles.

D'après les données ci-dessus, il en résulte que la masse gneissique glissante forme un dièdre tronqué, limité par les deux plans de discontinuité $\left(\mathrm{P}_{1}\right.$ et $\left.\mathrm{P}_{2}\right)$, par le plan $\left(\mathrm{P}_{3}\right)$ de la faille $\left(\mathrm{F}_{2}\right)$ en amont et par le talus oriental du monastère en aval (fig. 6). Par conséquent, on peut tracer sur la carte le contour superficiel du dièdre rocheux.

\subsection{Analyse des forces qui agissent sur le dièdre rocheux dêterminant}

L'interface $\left(\mathrm{P}_{3}\right)$ du dièdre tronqué et du massif du gneiss qui correspond au plan de faille - N $20^{\circ}-\mathrm{SE}$ $85^{\circ}$ - étant sensiblement verticale, il est évident qu'elle n'intervient pas dans le glissement du dièdre et n'a pas à être calculée dans l'analyse de sa stabilité.

Si le dièdre rocheux est détaché du massif de gneiss à deux micas, les valeurs de la résistance au cisaillement le long des interfaces $\left(\mathrm{P}_{1}\right),\left(\mathrm{P}_{2}\right)$ et $\left(\mathrm{P}_{3}\right)$, données par les cohésions correspondantes, doivent être nulles et par conséquent, seules les forces de frottement interne qui s'opposent au glissement du dièdre, interviennent.

Les forces qui agissent sur le dièdre sont d'une part son poids et, d'autre part, les pressions hydrostatiques développées.

Le poids total du dièdre est de l'ordre de $300000 \mathrm{kN}$ $\left(\mathrm{W}_{\mathrm{t}}=300000 \mathrm{kN}\right)$ et correspond à la somme de son poids propre $\left(\mathrm{W}_{\mathrm{d}}=250000 \mathrm{kN}\right)$ et du poids total des bâtiments du monastère $\left(\mathrm{W}_{\mathrm{b}}=50000 \mathrm{kN}\right)$. 
Les pressions hydrostatiques qui agissent perpendiculairement aux interfaces du dièdre, ont été déterminées approximativement à partir de la forme de la surface hydrostatique de l'eau dans une fouille creusée dans les gneiss à deux micas, dans le sous-sol du monastère. En supposant que la répartition des pressions interstitielles dans les surfaces de discontinuité est linéaire et que les pressions hydrostatiques sur l'interface $\left(P_{3}\right)$ sont nulles, les pressions hydrostatiques sur l'interface $\left(\mathrm{P}_{1}\right)$ ont été estimées à $\mathrm{U}_{1} \approx 30000 \mathrm{kN}$ alors que sur l'interface $\left(\mathrm{P}_{2}\right)$ elles ont été estimées à $\mathrm{U}_{2}$ $\approx 25000 \mathrm{kN}$

Pour l'analyse de la stabilité du diẽdre rocheux, les valeurs d'angle de frottement interne utilisées sont celles du tableau I. Plus précisément, pour l'interface $\left(\mathrm{P}_{1}\right)$ qui s'identifie avec le plan de stratification des gneiss à deux micas, la valeur de $23^{\circ}$ a été retenue pour des roches saturées d'eau, tandis que pour l'interface $\left(\mathrm{P}_{2}\right)$ qui recoupe le plan de stratification des gneiss à deux micas, la valeur de l'angle de frottement interne utilisée est $27^{\circ}$, correspondant à la roche saturée.

D'après ce qui précède et avec l'aide de la méthode des projections stéréographiques du canevas de Schmidt, le cône de frottement apparent du dièdre a été construit sur le diagramme stéréographique de la figure 7 .

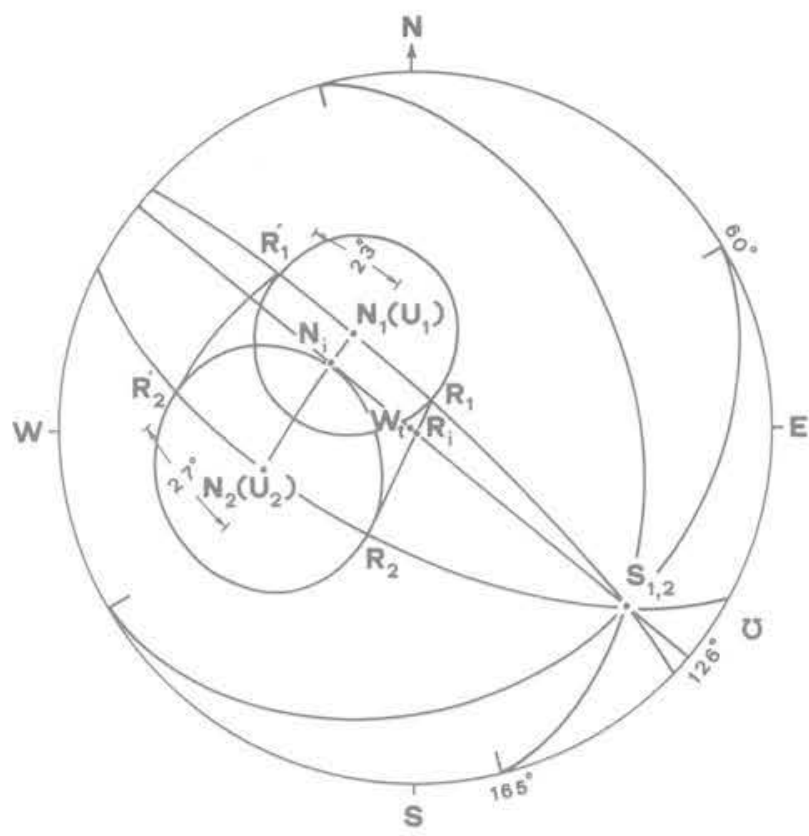

Fig. 7. - Cône de frottement sur le diagramme stéréographique de Schmidt.

Sur ce diagramme stéréographique, le point $W_{t}$ représente la position de la résultante des forces agissant sur le dièdre qui s'identifie en ce cas, avec le vecteur poids du dièdre. Le point $N_{1}=N_{1}+N_{2}$ représente la position de la résultante des forces normales sur les deux interfaces $\left(P_{1}\right)$ et $\left(P_{2}\right)$, tandis que le point $R_{1}=$
$\mathrm{R}_{1}+\mathrm{R}_{2}$ représente la résultante des forces de frottement interne agissant sur les interfaces. Enfin, l'angle entre $\left(\mathrm{N}_{\mathrm{f}}\right)$ et $\left(\mathrm{R}_{\mathrm{f}}\right)$ mesuré sur le grand cercle passant par les points $\left(S_{1}, 2\right)$ et $\left(W_{t}\right)$ représente l'angle de frottement apparent, c'est-à-dire l'angle au sommet du cône de frottement apparent.

D'après le diagramme stéréographique qui correspond aux conditions d'équilibre du dièdre pendant la période de sécheresse - période d'été où les pressions hydrostatiques sont négligeables - le dièdre est en équilibre, car son coefficient de sécurité, donné par l'expression ci-dessous, est supérieur à l'unité.

$$
F_{s}=\frac{\operatorname{tg}\left(N_{i}\right)\left(R_{i}\right)}{\operatorname{tg}\left(N_{i}\right)\left(W_{t}\right)}
$$

soit:

$$
F_{s}=\frac{\operatorname{tg} 24^{\circ}}{\operatorname{tg} 23^{\circ}}=\frac{0,445}{0,424}
$$

étant donné que $\left(\mathrm{N}_{\mathrm{i}}\right)\left(\mathrm{R}_{\mathrm{i}}\right)=24^{\circ}$ et $\left(\mathrm{N}_{\mathrm{t}}\right)\left(\mathrm{W}_{\mathrm{t}}\right)=23^{\circ}$

d'où :

$$
F_{s}=1,05
$$

Par contre, pendant la période d'humiditié - période d'hiver - les pressions hydrostatiques développées perpendiculairement aux deux interfaces du dièdre, ne sont plus négligeables et se composent avec le vecteur du poids $\left(W_{t}\right)$ du dièdre. La résultante de ces forces $\left(\mathrm{W}_{\mathrm{h}}=\mathrm{W}_{\mathrm{t}}+\mathrm{U}_{1}+\mathrm{U}_{2}\right)$, dont la grandeur se détermine à partir du polygone des forces de la figure 8 , n'est plus verticale et se place en dehors de la surface de stabilité $\left(R_{1}, R_{2}, R_{2}^{\prime}, R_{1}^{\prime}\right)$ du diagramme stéréographique de la figure 9 .

Sur le diagramme stéréographique, la position de la résultante des forces $\left(\mathrm{W}_{\mathrm{h}}\right)$ du dièdre se trouvant dans

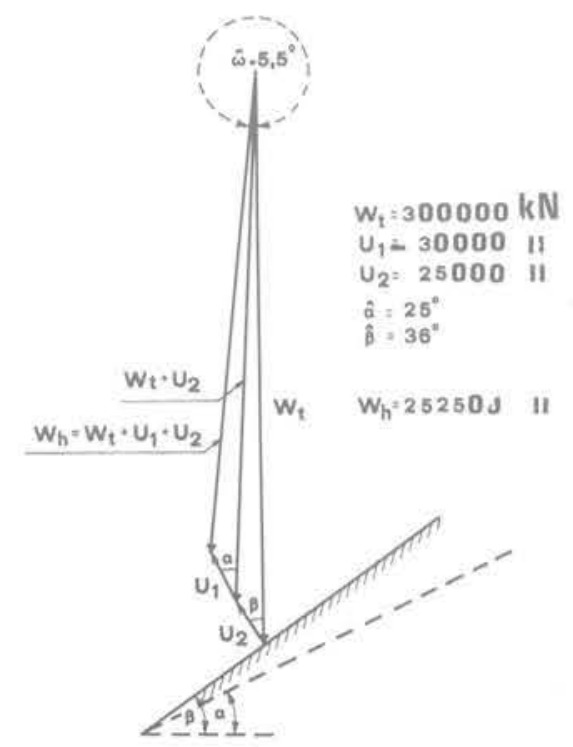

Fig. 8. - Equilibre des forces (régime d'hiver). 


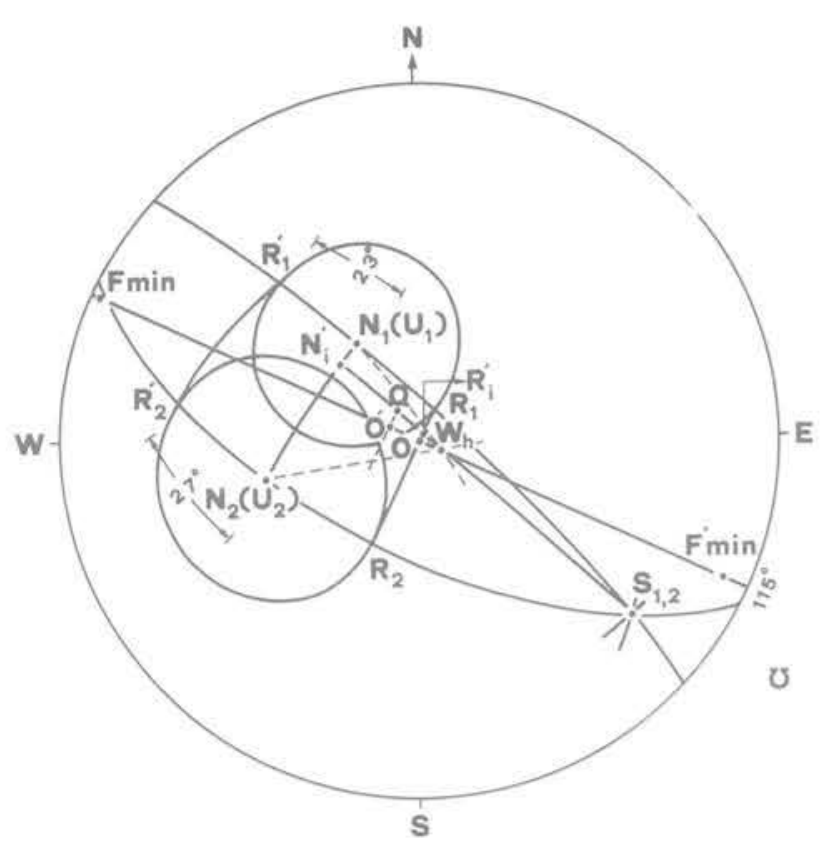

Fig. 9. - Diagramme stéréographique de Schmidt pour le calcul de $F_{\text {min }}$.

la surface qui se limite par les points $\left(R_{1}\right),\left(S_{1}, 2\right)$ et $\left(R_{2}\right)$, le glissement risque de se produire sur l'arête d'intersection $\mathrm{N} 126^{\circ}-\mathrm{SE} 23^{\circ}$ de deux interfaces du dièdre rocheux.

En ce cas, la valeur du coefficient de sécurité du dièdre se détermine à partir de l'expression suivante:

$$
F_{s}=\frac{\operatorname{tg}\left(N_{i}\right)\left(W_{h}\right)}{\operatorname{tg}\left(N_{i}\right)\left(R_{i}\right)}
$$

puisque :

$$
\left(N_{i}^{\prime}\right)\left(R_{i}^{\prime}\right)=25^{\circ} \text { et }\left(N_{i}^{\prime}\right)\left(W_{h}\right)=27^{\circ}
$$

on obtient:

$$
F_{s}=\frac{\operatorname{tg} 25^{\circ}}{\operatorname{tg} 27^{\circ}}=\frac{0,466}{0,509}
$$

d'oû:

$$
1_{s}=0,92
$$

A partir de ce qui précède, on voit que l'eau joue un rôle prépondérant dans le problème de la stabilité du dièdre rocheux sur lequel est fondé le compartiment oriental du monastère. En effet, les déplacements observés entre les épontes des fractures des murs des bâtiments, sont associés avec la période d'hiver, lorsque les gneiss à deux micas sont saturés d'eau et lorsque les pressions hydrostatiques développées dépassent une certaine limite.

\subsection{Forces nécessaires pour le maintien en équilibre}

Dans les conditions d'équilibre énoncées précédemment, le dièdre rocheux peut être en équilibre au moment où la position de la résultante des forces agissant $\left(W_{h}\right)$ intersecte la limite de la surface de stabilité $\left(\mathrm{R}_{1}, \mathrm{R}_{2}, \mathrm{R}_{2}^{\prime}, \mathrm{R}_{1}^{\prime}\right)$ du diagramme stéréographique. Le coefficient de sécurité du dièdre sera alors égal à l'unité $\mathrm{F}_{\mathrm{s}}=1$.

D'une manière concrète, avec l'aide d'une force extérieure minimale $\left(\mathrm{F}_{\min }\right)$ agissant sur le dièdre, il est possible de déplacer la position de la résultante des forces du dièdre sur la limite de la surface de stabilité du diagramme stéréographique à une position exacte car la distance entre le point $\left(W_{h}\right)$ et le grand cercle passant par les points $\left(R_{1}\right)$ et $\left(R_{2}\right)$ doit être minimale.

La grandeur et l'orientation de cette force minimum agissant sur un plan passant par les points $\left(W_{h}\right)$ et $(O)$, se déterminent à partir du polygone des forces de la figure 7 et avec l'aide du diagramme stéréographique de la figure 9 .

On a:

$$
\mathrm{F}_{\min }=\mathrm{W}_{\mathrm{h}} \cdot \sin \left(\mathrm{W}_{\mathrm{t}}\right)(\mathrm{O})
$$

puisque:

$$
\left(W_{t}\right)(O)=4^{\circ}
$$

on obtient:

$$
\mathrm{F}_{\min }=\mathrm{W}_{\mathrm{h}} \cdot \sin 4^{\circ}=252500 \times 0,069
$$

d'où :

$$
\mathrm{F}_{\min }=17610 \mathrm{kN}
$$

dont l'orientation est: N $115^{\circ}$-NW $2^{\circ}$

Etant donné que le gradient de séismicité de la péninsule du mont Athos est très élevé, il y a un risque de glissement du dièdre rocheux, malgré la présence de la force extérieure $\mathrm{F}_{\min }$, parce que les forces développées au cours d'une puissante secousse séismique peuvent déplacer la position de la résultante des forces agissant sur le dièdre, en dehors de la surface de stabilité du diagramme stéréographique.

Pour un coefficient de sécurité de l'ordre de $1,5\left(\mathrm{~F}_{\mathrm{s}}=\right.$ $1,5)$, qui peut donner au dièdre rocheux une stabilité assez élevée, même dans le cas d'une secousse séismique assez puissante, la nouvelle position (Q) de la résultante des forces agissantes sur le dièdre par rapport à la limite de la surface de stabilité du diagramme stéréographique peut être déterminée à partir de l'expression suivante:

$$
F_{s}=\frac{\operatorname{tg}\left(N_{i}\right)\left(R_{i}\right)}{\operatorname{tg}\left(N_{i}\right)(Q)}
$$

puisque:

$$
\operatorname{tg}\left(N_{i}\right)\left(R_{i}\right)=0,466 \text { et } F_{s}=1,5
$$

on obtient:

$$
\operatorname{tg}\left(N_{i}\right) \widehat{(Q)}=\frac{0,466}{1,500}=0,31
$$

d'où:

$$
\left(N_{i}\right)(Q)=17^{\circ}
$$




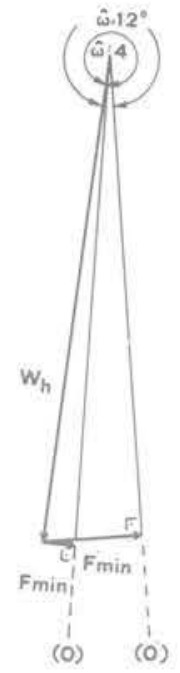

Fig. 10. - Polygone des forces pour le calcul de $F_{\text {min }}$.

La grandeur et l'orientation de la force nécessaire minimale $\left(\mathrm{F}_{\min }\right)$ qu'il est nécessaire d'appliquer sur le dièdre pour obtenir un coefficient de sécurité de l'ordre de $1,5\left(\mathrm{~F}_{\mathrm{s}}=1,5\right)$, peuvent être déterminées, cormme précédemment, à partir du polygone des forces de la figure 9. Cette force agit en effet, sur le plan passant par les points $\left(W_{h}\right)$ et $(O)$, et avec l'aide du diagramme stéréographique on a:

$$
F_{\text {min }}^{\prime}=W_{h} \cdot \sin \left(W_{h}\right)\left(O^{\prime}\right)
$$

puisque:

$$
\left(\mathrm{W}_{\mathrm{h}}\right)(\mathrm{O})=4^{\circ} \text { et }\left(00^{\prime}\right)=8^{\circ}
$$

on obtient:

$$
F_{\min }=W_{h} \cdot \sin 12^{\circ}=252500 \times 0,207
$$

d'oû:

$$
\mathrm{F}_{\text {min }}^{\prime}=52270 \mathrm{kN}
$$

donc son orientation est: $\mathrm{N} 115^{\circ}-\mathrm{E} 6,5^{\circ}$

Cette force extérieure minimale de quelque $52000 \mathrm{kN}$ devrait être appliquée sur le dièdre rocheux sous forme de barres d'acier scellées sur toute leur longueur par un mortier ou par des résines synthétiques d'orientation N $115^{\circ}-\mathrm{SE} 6^{\circ}$. Pour garantir sa stabilité, il faudra qu'elle soit répartie sur une grande surface de la masse gneissique glissante.

\section{CONCLUSION}

Le déplacement du compartiment oriental du monastère de Stauronikita qui s'accompagne par un grand nombre des fractures sur les murs des bâtiments, est dû au glissement d'un dièdre tronqué des "gneiss à deux micas» sous-jacents.
Pendant la période de sécheresse où les pressions hydrostatiques sont négligeables, on peut estimer que le dièdre rocheux est en équilibre $\left(F_{s}=1,05\right)$.

Par contre, pendant la période hivernale, où interviennent les pressions hydrostatiques, le coefficient de sécurité est inférieur à l'unité $\left(\mathrm{F}_{\mathrm{s}}<1\right)$ et le glissement risque de se produire sur l'arête d'intersection - $\mathrm{N}$ $126^{\circ}$-SE $26^{\circ}$ - de deux interfaces $\left(P_{1}\right)$ et $\left(P_{2}\right)$ du dièdre. Dans ce cas, la force nécessaire minimale $\left(\mathrm{F}_{\min }\right)$ qu'il faudrait appliquer sur le dièdre rocheux pour obtenir un coefficient de sécurité égal à l'unité $\left(F_{\mathrm{s}}=1\right)$ est de l'ordre de $17600 \mathrm{kN}$, avec une orientation $\mathrm{N}$ $115^{\circ}-\mathrm{NW} 2^{\circ}$.

Enfin, pour un coefficient de sécurité de l'ordre de 1,5 $\left(\mathrm{F}_{\mathrm{s}}=1,5\right)$ qui mettrait en sûreté la stabilité du dièdre rocheux, même dans le cas d'une secousse séismique assez puissante, la grandeur de la force nécessaire minimale $\left(\mathrm{F}_{\min }\right)$ qu'il faudrait appliquer sur le dièdre est de l'ordre de $52300 \mathrm{kN}$, avec une orientation $\mathrm{N}$ $115^{\circ}$-SE $6^{\circ}$.

\section{BIBLIOGRAPHIE}

BARTON, N.R. - Estimating the shear strength of Rock Joits. - Proceedings of the 3rd. Congr. Int. Soc. Rock Mech. Vol. 2, 1974.

DEMIRIS, C.A - Géologie du génie civil. - Thessaloniki, 1979.

HOEK, E. ; BRAY, J.W. - Rock slope engineering. Revised second edition, Institution of Mining and Metallurgy, London, 1977.

HORN, H.M. ; DEERE, D.U. - Frictional characteristics of minerals geotechnique. - Vol. 12, $\mathrm{n}^{\circ} 4$, 1962.

KENNEY, T.C. - The influence of mineral composition of the residual strength of natural soils. Proceedings Geotechnical Conference, Oslo, vol. 1. 1967.

KOCKEL, F.; MOLLAT, H. ; WALTHER, H. Geologie des Serbo-Mazedonischen Massius und seines mesozoischen Rahmens (Nord-griechenland). - Geol. Jb, 89, 1971.

MATTAUER, M. - Les déformations des matériaux de l'écorce terrestre. - Herman Ed. Paris, 1973.

MERCIER, J. - Principal results of a geotectonic study of the Aegean Arc and its localisation within the Eastem Mediterranean. - VI Col. Geol. Aeg. Reg, Vol. III, 1977, pp. 1281-1291.

PANET, M; ROTHEVAL, J.-P. - Stabilité des talus rocheux. - Bull. Liaison Labo. P. et Ch., $n^{\circ}$ spécial décembre 1976.

RAMSAY, J.G. - Folding and Fracturing of rocks. Mc Graw Hill, Ed. London, 1967.

VIALLON, R.; RUHLAND, M.; GROLIER, J. Eléments de tectonique analytique. - Masson Ed. Paris, 1976. 